\title{
A Promising Listeria-Vectored Vaccine Induces Th1-Type Immune Responses and Confers Protection Against Tuberculosis
}

\begin{abstract}
Yuelan Yin ${ }^{1 *}$, Kai Lian ${ }^{1}$, Dan Zhao ${ }^{1}$, Chengwu Tao ${ }^{1}$, Xiang Chen ${ }^{1}$, Weijun Tan ${ }^{1}$, Xiaobo Wang ${ }^{1}$, Zhengzhong $\mathrm{Xu}^{1}$, Maozhi Hu ${ }^{1}$, Yan Rao ${ }^{2}$, Xiaohui Zhou ${ }^{3}$, Zhiming Pan ${ }^{1}$, Xiaoming Zhang ${ }^{1,4}$ and Xin'an Jiao ${ }^{1 *}$

1 Jiangsu Key Laboratory of Zoonosis, Joint International Research Laboratory of Agriculture and Agri-Product Safety, Jiangsu Co-Innovation Center for Prevention and Control of Important Animal Infectious Disease and Zoonosis, Yangzhou University, Yangzhou, Yangzhou, China, ${ }^{2}$ ABSL-3 Lab, Wuhan University, Wuhan, China, ${ }^{3}$ Department of Pathobiology and Veterinary Science, University of Connecticut, Storrs, CT, United States, ${ }^{4}$ Institut Pasteur of Shanghai, Chinese Academy of Sciences, Shanghai, China
\end{abstract}

Deaths associated with tuberculosis (TB) is rising and accounted for 1.4 million deaths in 2015 many of which were due to drug-resistant bacteria. Vaccines represent an important medical intervention, but the current Bacilli Calmette-Guerin (BCG) vaccine is not ideal for the protection of teenagers and adults. Therefore, a safe and effective

OPEN ACCESS

Edited by:

Dongsheng Zhou,

Beijing Institute of Microbiology and Epidemiology, China

Reviewed by:

Sukhadeo B. Barbuddhe, ICAR-National Institute of Biotic Stress

Management, India

Hongjie Fan,

Nanjing Agricultural University, China

*Correspondence:

Yuelan Yin

yylan@yzu.edu.cn

Xin'an Jiao

jiao@yzu.edu.cn

Received: 19 June 2017 Accepted: 04 September 2017 Published: 28 September 2017

Citation

Yin Y, Lian K, Zhao D, Tao C, Chen X, Tan W, Wang X, Xu Z, Hu M, Rao Y, Zhou $X$, Pan Z, Zhang $X$ and Jiao $X$ (2017) A Promising Listeria-Vectored Vaccine Induces Th1-Type Immune Responses and Confers Protection Against Tuberculosis.

Front. Cell. Infect. Microbiol. 7:407. doi: 10.3389/fcimb.2017.00407 vaccine is urgently needed. In this study, we designed a novel vaccine using an attenuated Listeria monocytogenes strain carrying fusion antigen FbpB-ESAT-6 (rLM) and characterized its safety and protective efficacy against Mycobacterium tuberculosis (M.tb) infection in mice. Compared to the wild type strain yzuLM4 and parental strain $L M \triangle a c t A / p / c B$ (LM1-2), the virulence of rLM was significantly reduced as judged by its infectious kinetics and $L D_{50}$ dose. Further characterization of intravenous immunization showed that prime-boost vaccination significantly increased the levels of Th1 cytokines (IFN- $\gamma$, IL-17, and IL-6), and enhanced cytotoxic T lymphocyte (CTL) CTLs activity, suggesting that rLM could elicit potent Th1/Th17 responses. More importantly, rLM significantly conferred the protection against M.tb H37Rv challenge. Collectively, our findings indicated that rLM is a novel and useful tool to prevent M.tb infection, and can be potentially be used to boost BCG-primed immunity.

Keywords: Mycobacterium tuberculosis, Th1/Th17, protective efficacy, Listeria monocytogenes, attenuated, FbpB-ESAT-6

\section{INTRODUCTION}

Tuberculosis (TB) is an infectious disease caused by the Mycobacterium tuberculosis (M.tb) complex. TB incidence has fallen by an average of $1.5 \%$ per year since 2000 , yet during the past few years, the proportion of multidrug-resistant TB (MDR-TB) and extensively drug-resistant TB (XDR-TB) cases has increased steadily (World Health Organization, 2016). Therefore, it is highly urgent to control TB occurrence. However, effective prevention of the infection, recurrence and reactivation of latent $\mathrm{TB}$ remain to be significant challenge. Vaccination is an important medical intervention in TB prevention strategies. Bacilli Calmette-Guerin (BCG) is the only vaccine approved worldwide for clinical use to prevent TB, but it fails to induce enough $M$. $t b$-specific CD8 ${ }^{+}$ 
T cells and elicit optimal immune memory in the lung (Beverley et al., 2014). Its efficacy is variable, ranging from 0 to $80 \%$ in different regions, and it is not ideal for the protection of teenagers or adults (Henao-Tamayo et al., 2014). TB vaccine developers are seeking new ways to rationally and efficiently select candidates for human efficacy trials.

Host immune response against $M . t b$ is mainly mediated by cellular immunity, in which Th1 cytokines IFN- $\gamma$, TNF- $\alpha$ are absolutely required to control bacterial growth (Cavalcanti et al., 2012; Kim et al., 2014). IL-17 secreted by Th17 cells can accelerate the initial response and promote the recruitment of Th1 cells to the site of infection, contributes to vaccine efficacy (Torrado and Cooper, 2010). IL-6 contributes to host resistance by its proinflammatory activity and by its influence on cytokine secretion. While anti-inflammatory cytokine IL-4 and IL-10 secreted by Th2 CD4 cells down-regulate the Th1 response, lead to pulmonary fibrosis (Rook et al., 2004). Effective TB vaccines will need to enhance Th1/Th17 immunity and suppress pre-existing Th2-like activity. Attenuated Listeria monocytogenes (LM), as an immune modulator and vaccine adjuvant, induces a strong cellular immunity characterized by Th1-type CD4 T cell and CD8 $\mathrm{T}$ cell activation, has been successfully developed and used as a vaccine carrier and is a attractive TB vaccine vehicle for delivering $M$.t $b$ antigens.

Because LM has a unique intracellular parasitic life (Decatur and Portnoy, 2000), it can be used as a promising adjuvant to induce the generation of innate immunity, through the secretion of various critically important cytokines, e.g., IFN- $\gamma$, IL-12, and IL-18 (Nomura et al., 2002), and the generation of adaptive immunity by preferentially promoting the proliferation of antigen-specific CD4 ${ }^{+} \mathrm{T}$ cells and $\mathrm{CD} 8^{+} \mathrm{T}$ cells. The secreted protein listeriolysin $\mathrm{O}$ (LLO) encoded by hly is prominent in generating the Th1 immune response (Yamamoto et al., 2005; Kono et al., 2012). Notably, Grode et al. successfully expressed LLO in BCG $\Delta$ ureC::hly (VPM1002), leading to superior protective efficacy (Grode et al., 2005). Gunn et al. fused LLO with HPV 16 E7 protein (Lm-LLO-E7), which can augment the E7-specific $\mathrm{CD}^{+} \mathrm{T}$ cell response and repressing murine tumors (Gunn et al., 2001). We have reported that the LLO-esat6 fusion protein delivered by attenuated LM significantly elicited a strong antigen-specific T cell-mediated immune response (Yin et al., 2012).

The safety of LM vaccine has been well-demonstrated in human clinical trials: Angelakopoulos's clinical trial demonstrated the ability of an attenuated LM-based empty vaccine to safely and effectively induce antigen-specific $\mathrm{T}$ cell responses in humans (Angelakopoulos et al., 2002); Maciag's Phase I trial and Petit's Phase II clinical trial with LM-LLO-E7 verified that the safe and effective recombinant vaccines could be potentially a new therapeutic option (Maciag et al., 2009; Petit and Basu, 2013). LM has particular advantages to offer as a neonatal vaccine vehicle: attenuated strains of LM that are safe for neonates have now been identified, and interestingly, they are very efficient at inducing robust Th1-type immunity in neonates (Kollmann et al., 2007).

LM has been successfully developed as a novel live vaccine vector, especially for tumor immunotherapy. However, LM-based
anti-M.tb vaccines are rarely reported. Both our previous report (Yin et al., 2012) and other studies (Miki et al., 2004; Lin et al., 2015) suggested that a Listeria-based vaccine could potentially prevent TB. In this study, protective antigens ESAT- 6 and FbpB with multiple $\mathrm{T}$ cell epitopes and $\mathrm{B}$ cell epitopes were chosen as delivering targets (D'Souza et al., 2003; Brodin et al., 2006), the fusion protein FbpB-ESAT-6 was expressed and characterized the innate and adaptive immune response induced by Listeria-based TB vaccine and determined its protective efficacy against wild type $M . t b$ challenge.

\section{MATERIALS AND METHODS}

\section{Bacteria}

Virulent serotype 1/2a LM strain yzuLM4 and attenuated LM strain yzuLM4 $\triangle a c t A / p l c B$ (LM1-2) were cultured in brain heart infusion (BHI) broth (Yin et al., 2008). M.tb H37Rv was preserved in an ABSL-3 lab at Wuhan University. BCG vaccine was purchased from the Yangzhou Center for Disease Control and Prevention.

\section{Experimental Animals}

Six-week-old female C57BL/6 mice $\left(\mathrm{H}-2^{\mathrm{b}}\right)$ were purchased from Vital River Laboratory Animal Technology Co., Ltd. (Beijing, China). All animals were immunized at the animal biosafety facilities and all procedures were approved by the institutional animal ethics approval committee of Yangzhou University. Animals received free access to water and commercial mouse chow throughout the study. The challenge experiments were conducted in an ABSL-3 lab at Wuhan University.

\section{Construction of the Recombinant Strain}

To achieve homologous recombination, the recombinant plasmid pKSV7-actA/fbpB-esat-6/plcB was constructed (Figure $\mathrm{S} 1$ ) with primers pairs showing in Table S1, in which the $f b p B$ gene (850 bp) was flanked with a DNA fragment consisting of the 698-bp upstream fragment of $a c t A$ and signal peptide sequence of actA (75 bp). The esat-6 gene was flanked by the downstream sequence of the $p l c B$ gene (861bp). A 30-bp linker encoding (Gly4Ser) was designed between $f b p B$ and esat-6 (285 bp). The recombinant plasmid pKSV7-actA/fbpBesat-6/plcB was electroporated into competent LM1-2 cells. The recombinant strain LM::fbpB-esat-6 (rLM) was obtained according to previously described methods (Watanabe-Takano et al., 2014). The positive clones were verified by sequencing.

\section{Western Blotting}

Secreted proteins were precipitated from culture supernatants using trichloroacetic acid (TCA) and run on a sodium dodecyl sulfate polyacrylamide gel. After transfer to a polyvinylidene difluoride (PVDF) membrane (NEN Life Sciences, Boston, MA) using a Western blot apparatus (Bio-Rad, Melville, NY), the proteins were incubated with a rabbit anti-ESAT-6 monoclonal antibody (HYB-076-08, Abcam, Hong Kong, China) and then stained with a peroxidase-labeled anti-rabbit antibody (BD Pharmingen, USA). 


\section{Virulence of Listeria Strains in Vivo}

The $\mathrm{LD}_{50}$ of listerial strains was determined with groups of mice intravenously inoculated with $0.1 \mathrm{~mL}$ of an appropriate threefold dilution of the recombinant or parent strain in PBS. Surplus bacteria were plated on BHI agar plates for bacterial counts. The mice were monitored for the next 14 days, and the median lethal dose $\left(\mathrm{LD}_{50}\right)$ was determined. Bacterial enumeration in spleens and livers of mice was evaluated via intravenously injected with $0.1 \mathrm{LD}_{50}$ of rLM or LM1-2 on day 1, 3, 5, and 7.

\section{Cytokine Secretion Assay}

Six-week-old C57BL/6 mice were randomly divided into five groups (five mice/group). Three groups were prime-boost immunized i.v. with of rLM (0.1LD50), LM1-2 (0.1LD50), and $100 \mu l$ PBS, respectively; the fourth group was immunized with BCG $\left(1 \times 10^{6} \mathrm{CFU}\right)$ i.v.; the fifth group was primely immunized with BCG $\left(1 \times 10^{6} \mathrm{CFU}\right)$, and boosted with rLM (0.1LD50). A total of $1 \times 10^{6}$ murine splenic cells lymphocytes from C57BL/6 mice were added onto 96-well microplates, The stimulator ESAT- $6_{1-20}$ and FbpB $_{240-259}$ (final concentration $5 \mu \mathrm{g} / \mathrm{ml}$ ), purified protein derivative (PPD, Switzerland Prionics company, final concentration $10 \mu \mathrm{g} / \mathrm{ml}$ ), and ConA (final concentration $10 \mu \mathrm{g} / \mathrm{ml}$ ) was co-incubated with these lymphocytes, respectively. Supernatants from each group were harvested and detected by mouse inflammation kit and mouse Th1/Th2/Th17 cytokine kit (R\&D, USA) according to manufacturers' instructions.

\section{Cytotoxicity Assay Using the CFSE Fluorescent-Based Dye}

To determine the cytotoxicity in vivo, the same immunization procedure was used as described as cytokine secretion assay. On day 8 after boost immunization, spleen cells were pooled from naive C57BL/6 mice and divided into two groups; the cell suspension for one group was incubated with PPD at $37^{\circ} \mathrm{C}$ for $45 \mathrm{~min}$ and subsequently labeled with $\mathrm{CFSE}^{\text {high }}(2.5 \mu \mathrm{M}$, Invitrogen, USA) buffer at $37^{\circ} \mathrm{C}$ for $10 \mathrm{~min}$, the other group was incubated without PPD and labeled with CFSE $^{\text {low }}(0.25 \mu \mathrm{M})$ buffer, $1 \times 10^{7}$ mixed cells were intravenously injected into mice that had previously prime-boost immunized with rLM, LM12, BCG, and PBS, respectively, and $15 \mathrm{~h}$ later, cell suspensions from spleen of mice were analyzed by flow cytometry, and each population was analyzed by a FACScan in which each population was detected according to the differing CFSE fluorescence intensities. To calculate specific lysis, the following formula was used: The percentage of specific lysis was calculated using the following formula: percent specific lysis $=100-[100 \mathrm{x}$ (\% $\mathrm{CFSE}_{\text {high }}$ immunized/\% $\mathrm{CFSE}_{\text {low }}$ immunized $) /\left(\% \mathrm{CFSE}_{\text {high }}\right.$ control/\% CFSE low $_{\text {control)]. }}$

\section{M.tb Challenge}

For challenge experiments, 6-week-old female C57BL/6 mice were randomly divided into five groups (eight mice/group), four groups were prime-boost immunized at 14 days interval, including PBS group $(100 \mu \mathrm{l})$, LM1-2 group $\left(1 \times 10^{6} \mathrm{CFU}\right)$, rLM $\left(2 \times 10^{7} \mathrm{CFU}\right), \mathrm{BCG} / \mathrm{rLM}$ group (primary vaccinated with BCG, boosted with rLM). The fifth group was BCG single immunized group $\left(1 \times 10^{6} \mathrm{CFU}\right)$. Twenty-eight days after second immunization, mice were challenged with $5 \times 10^{5} \mathrm{CFU}$ virulent M.tb $\mathrm{H} 37 \mathrm{Rv}$ via a lateral tail vein, the inoculum plated at the time of challenge. Six weeks post-challenge, all mice were sacrificed. The spleens and livers of five mice per group were removed, homogenized and cultured for CFU of M.tb. The left lung of each mouse was excised and fixed in $10 \%$ phosphate-buffered formalin, sectioned, stained with hematoxylin and eosin, and examined for histological lesions with microscope. Alternatively, the tissues were subjected to acid-fast staining to visualize the bacilli. The challenge experiment was conducted twice.

\section{Statistical Analysis}

Differences between groups were analyzed using the Statistical Package for Social Sciences software (SPSS version 15.0; SPSS, Chicago, IL, USA). $P<0.05$ were considered significant, and values $<0.01$ were considered highly significant.

\section{RESULTS}

\section{Expressed Fusion Protein FbpB-ESAT-6 Retains Hemolytic Activity and Immunogenicity}

A recombinant L. monocytogenes strain LM::fbpB-esat-6 was successfully constructed via homologous recombination technology, the secretive expression of FbpB-ESAT- 6 was verified by Western blotting using rabbit anti-ESAT-6 monoclonal antibody. The appearance of the specific $36 \mathrm{kDa}$ target band indicated that fusion protein Ag85B-ESAT-6 secretively expressed and had an effective immunoreactivity (Figure 1A). LLO provides LM unique adjuvant character of generating Th1 immune response, the hemolysis activity of rLM was performed, as shown in (Figure 1B), the hemolysis titer of the recombinant strains reached $2^{3}$, which was lower than that of the parental strains yuzLM4 $\left(2^{4}\right)$, thus rLM displayed good hemolytic activity.

\section{The Virulence of Recombinant LM Strain was Reduced in Mice}

Novel vaccine candidates are aimed at prevented human tuberculosis, the safety of rLM is firstly considered and evaluated by bacterial growth kinetics and $\mathrm{LD}_{50}$ determination. The bacterial growth kinetics in vivo showed that there was no significant difference in livers and spleens of mice in rLM infection group comparing with LM1-2 group $(P>0.05)$ on each detection point, indicated that the translocation ability of the recombinant strain was reduced and the virulence is decreased (Figures 1C,D). The notable reduction of virulence was shown in Table 1, the recombinant strain was reduced by $4.67 \times 10^{4}$ - and 4.9 -fold comparing with wild type strain yzuLM4 and its parental strain LM1-2, respectively. These results suggested that the safety of the recombinant strain was distinctly improved.

\section{Pro- and Anti-inflammatory Cytokine Responses among Groups}

Pro-inflammatory cytokine IFN- $\gamma$ is crucial for the immune response to $M . t b$, and IL-17 contributes antibacterial infection. 
A

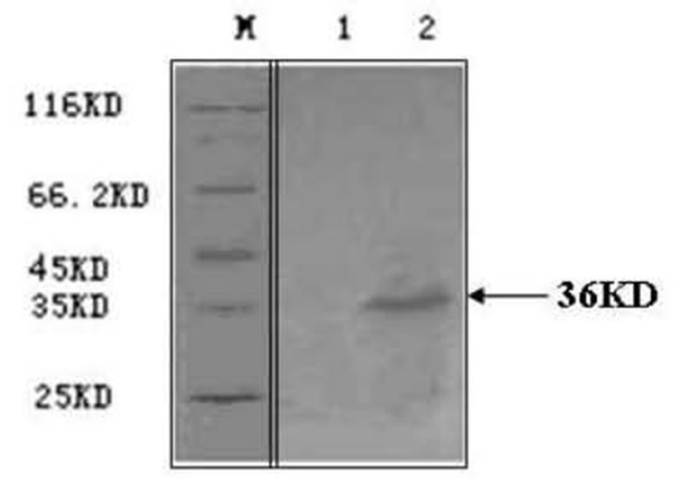

B

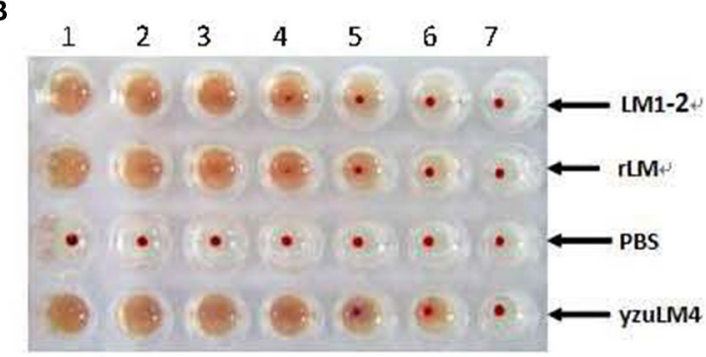

C
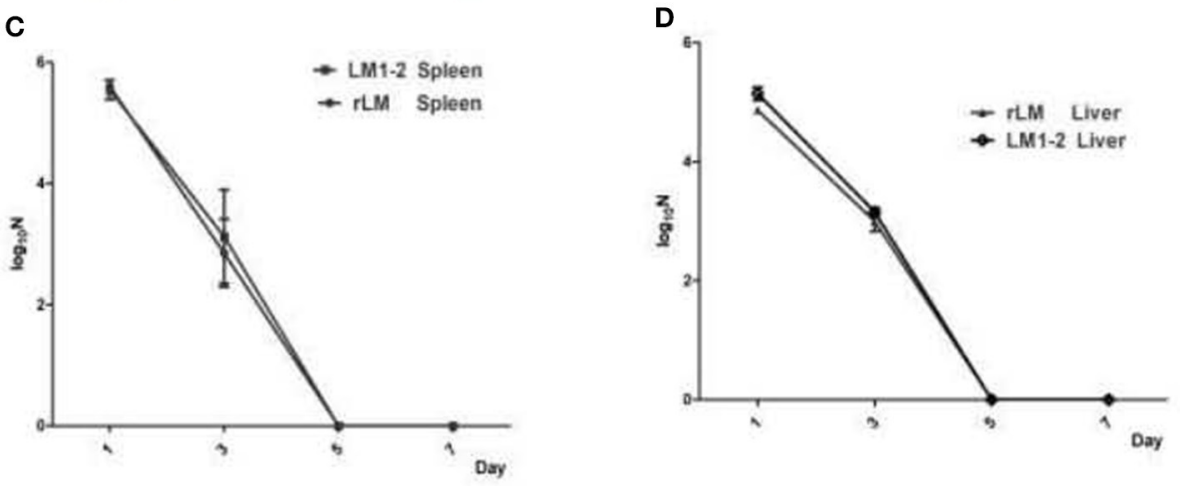

FIGURE 1 | Identification of recombinant L. monocytogenes strain. (A) Western blot analysis of expressed FbpB-ESAT-6 protein. Secreted proteins were TCA-precipitated and probed by a rabbit anti-ESAT-6 monoclonal antibody by western blot. M represents protein marker; lane 1 represents proteins from LM1-2, lane 2 represents the proteins from rLM. (B) Hemolytic activity of listeria strains. Culture supernatants were incubated with sheep erythrocytes at $37^{\circ} \mathrm{C}$ in two-fold serial dilutions (wells $1-7$ ). The recombinant strain $r L M$ had hemolysis titers of $2^{3}$, while the parental strains were $2^{4}$. (C,D) Kinetics of different $L M$ strains during infection in spleens (C) and livers (D) of mice. C57BL/6 mice (20 mice per group) were injected intravenously (i.v.) with rLM and LM1-2 at a dose of 0.1 LD 50 , respectively. Bacteria in spleen and liver were enumerated on day $1,3,5$, and 7 post-inoculation by tissue homogenization and serial plating.

TABLE 1 | LD 50 of recombinant, parental, and wild type strains.

\begin{tabular}{|c|c|c|c|c|c|c|c|c|c|c|c|c|}
\hline \multirow{2}{*}{$\begin{array}{l}\text { Dose (CFU) } \\
\text { CFU per mouse }\end{array}$} & \multicolumn{4}{|c|}{ yzuLM4 $\left(1 \times 10^{4}\right)$} & \multicolumn{4}{|c|}{ LM1-2 $\left(1 \times 10^{7}\right)$} & \multicolumn{4}{|c|}{$\operatorname{rLM}\left(1 \times 10^{8}\right)$} \\
\hline & 15.03 & 5.01 & 1.67 & 0.56 & 10.53 & 3.51 & 1.17 & 0.39 & 29.3 & 9.75 & 3.25 & 1.08 \\
\hline Mortality & $5 / 5$ & $5 / 5$ & $5 / 5$ & $2 / 5$ & $5 / 5$ & $1 / 5$ & $0 / 5$ & $0 / 5$ & $5 / 5$ & $5 / 5$ & $3 / 5$ & $0 / 5$ \\
\hline
\end{tabular}

C57BL/6 mice (five mice per group) were injected i.v. with increasing doses of recombinant and parental strains. The mice were monitored for the next 14 days and data were calculated by the Reed-Muench method.

The results illustrated in Figure 2 showed that significantly higher levels of IFN- $\gamma$ and IL-17A were induced by vaccination with rLM than the negative control group $(P<0.01 ; P<0.05)$ and BCG group $(P<0.01 ; P<0.05)$ in spleens (Figures $2 \mathrm{~A}, \mathbf{B}$ ), significantly higher levels of TNF- $\alpha$ and IL-6 (Figures 2C,D) than the negative control group $(P<0.05)$; while low level of IL4 were induced in each group (Figure 2E), and there was no significant difference among groups except PPD as stimulator. 
These results indicated that Th1/Th17 cellular immunity was elicited by rLM.

\section{rLM Strain Elicited Strong CTL Immune Responses}

CTL plays a very important role in cellular immune responses, conducing to eradicate of intracellular paracitical M. tuberculosis. Mice immunized with rLM were able to produce efficiently PPD-specific cytotoxic $\mathrm{T}$ lymphocytes than the negative control group $(P<0.05)$ (Figure 3). Additionally, the killing effect of the group with combined immunization of both BCG and rLM (BCG/rLM) was stronger than BCG immunized group $(P<0.01)$. These results indicated that rLM strain could elicite strong CTL immune responses against $M . t b$ antigens. The strong CTL immune responses elicited by rLM were predicted to enhance the clearance of M.tb.

\section{Protective Efficacy of Prime-Boost rLM Enhanced Protection by BCG Prime-rLM Boost}

The vaccination and challenge procedure was shown in Figure 4A. The result of immune protective efficacy showed that two mice died in the PBS group on day 14 and 28, respectively, and one mouse died on day 28 in the LM1-2 vaccination group. In the other three groups, all the mice survived, and the protective efficacy of these three groups was 100\% (Figure 4A).

\section{Reduced Pathology Following Prime-Boost with rLM}

The bacterial load and histopathological assays suggested that the prime-boost rLM not only reduced the bacterial load, but also conferred superior protection against pathological damage and granuloma formation (Figures 4B,C). It was interesting that the LM1-2-vaccinated group reduced granuloma inflammation in the lung by $10.5 \%$ (Figure $4 \mathrm{~B}$ ). The combination vaccine BCG prime-rLM boost strategy showed superior protection against TB.

\section{Decreased Bacterial Load in the Lung}

The prime-boosted rLM group significantly reduced the bacterial load in the lung compared with LM1-2 group $(p<0.01)$. The bacterial load in the lungs of mice vaccinated with $\mathrm{BCG} / \mathrm{rLM}$ was significantly lower than in the BCG group $(p<0.01)$ (Figures 4D,E). The Bacilli observed in tissues subjected to acidfast staining was consistent with bacterial load calculation in the lung and spleen (Figure 4F). Thus, these results suggested that rLM could confer protection against $M$.tb challenge.

\section{DISCUSSION}

TB is a serious worldwide threat to public health security, and it is urgent to design and develop novel TB vaccines. Novel approaches to $\mathrm{TB}$ vaccination are developing rapidly, and 17 vaccine candidates are in the clinical trial (Garg et al., 2014). However, how to enhance the synergistic action between $\mathrm{CD}^{+}$- and $\mathrm{CD}^{+}{ }_{-}^{-\mathrm{T}}$ cells remains worth exploring (Principi and Esposito, 2015; Shang et al., 2016). LM as an intracellular pathogenic antigen carrier, has the advantage of inducing multivalent innate immunity as well as a cell-mediated immune

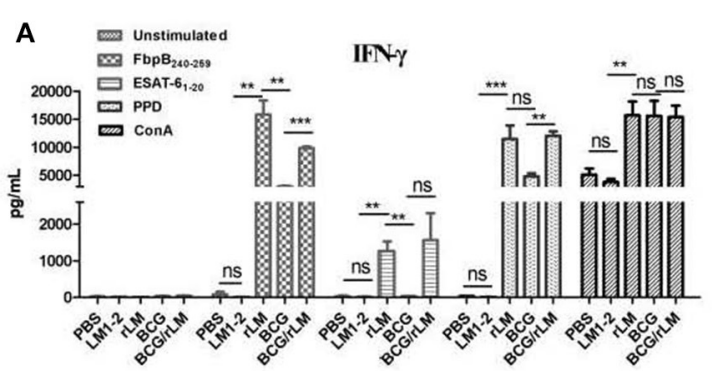

B
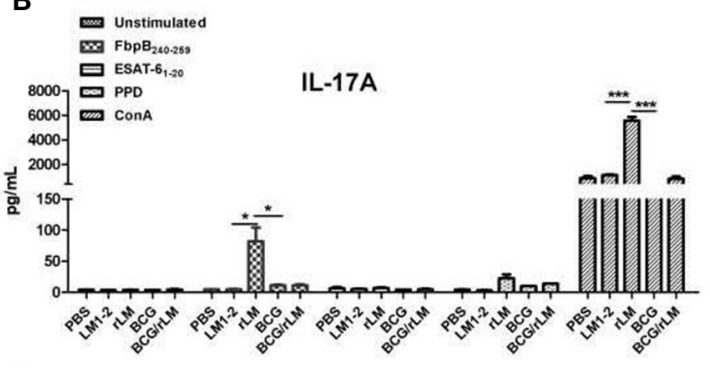

。
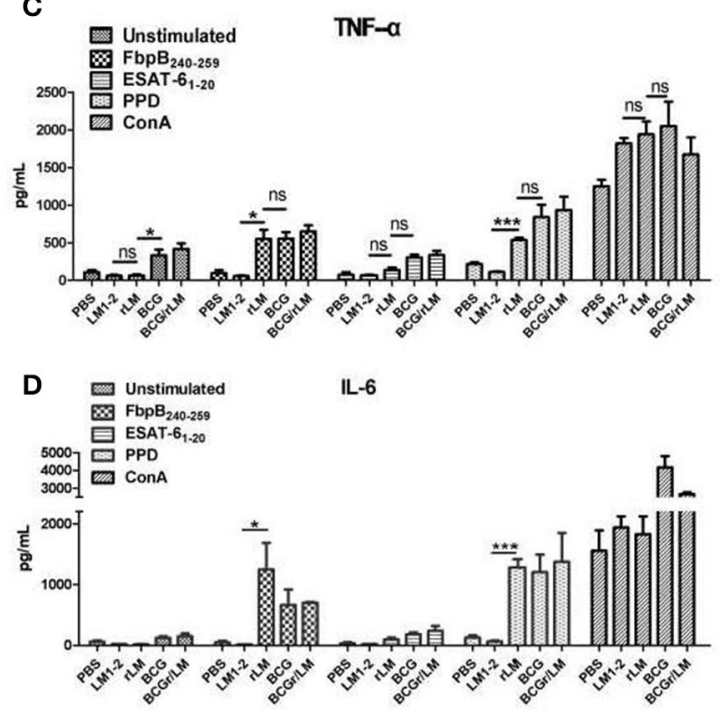

E

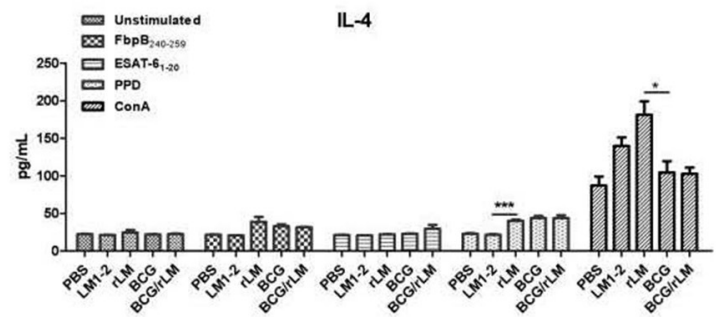

FIGURE 2 | The level of inflammatory cytokines in the splenocytes of mice immunized with indicated LM strains or controls. Five groups of C57BL/6 mice $(n=5)$ were intravenously immunized with $r L M, L M 1-2, P B S, B C G$, and BCG/rLM, respectively. IFN- $\gamma$ (A), IL-17A (B), TNF- $\alpha$ (C), IL-6 (D), and IL-4 (E) were determined on the 8th day after immunization. Splenocytes were prepared and stimulated with peptide $\mathrm{FbpB}_{240}-259$, ESAT-6 $1-20$, PPD and ConA. Cytokines in the supernatants from each well were determined by ELISA. The results were presented as the mean $\pm \mathrm{SD}$ of three wells in triplicate. ${ }^{\star * \star}$ indicated $P<0.001$, ${ }^{* \star}$ indicated $P<0.01$, and ${ }^{*}$ indicated $P<0.05$ of the comparisons. (A-E) Cytokines in the splenocytes. 

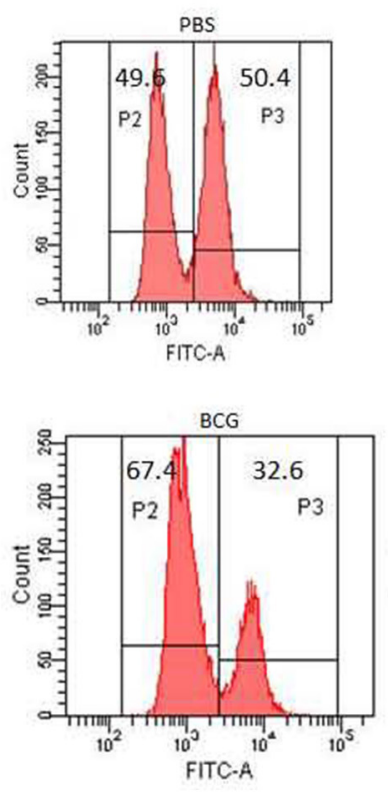
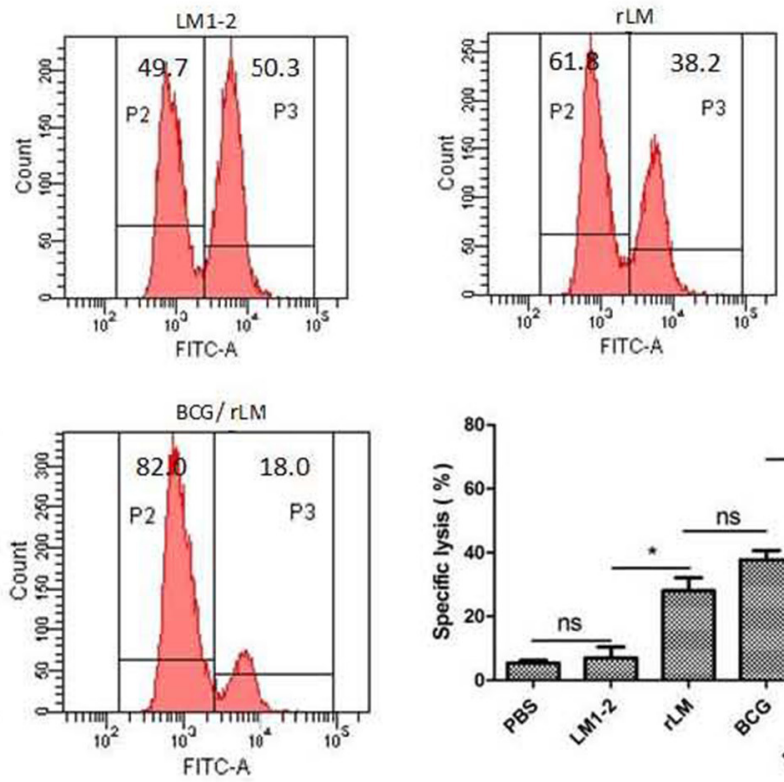

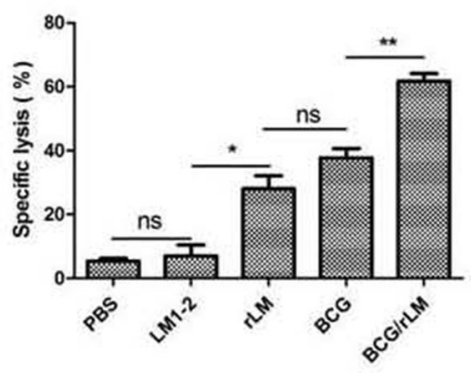

FIGURE 3 | PPD-specific CTL responses in vivo. PPD-specific CTL activity in vivo in mice ( $n=5$ per group) immunized with rLM, LM1-2, PBS, BCG, or BCG/rLM, respectively. A mixture of CFSE-labeled splenocytes pulsed with PPD (CFSE ${ }^{\text {high}}$ ) or without PPD(CFSElow) was intravenously injected into each group of mice on day 7 after the second immunization. The histogram shows CFSElow untreated and CFSEhigh PPD pulsed target cells in the spleens of mice after 15 h of transfer, respectively. Results were representative of three experiments, each with five mice per group. ${ }^{\star} P<0.05$ rLM group vs. LM1-2 group; ${ }^{\star \star} P<0.01$ BCG/rLM group vs. BCG group.

response (Jiang et al., 2007). The advantage of LM inducing strong protective immune responses has been intensively studied in molecular biology and immunology (Condotta et al., 2012). It is an effective and promising vaccine vehicle for tumor immunotherapy. Based on the safety and immunity investigation of our previous study on LM::esat-6, the vector potentiality of the highly attenuated LM for delivering the M.tb antigens FbpB and ESAT-6 was further probed.

It is worth mentioning that $\mathrm{CD}^{+} \mathrm{T}$ cells are believed to play a central role in the control and obliteration of intracellular pathogens (Agnellini et al., 2008; Condotta et al., 2012). The major functional subset of $\mathrm{CD}^{+} \mathrm{T}$ cells is $\mathrm{CD}^{+}$cytotoxic lymphocytes (CTL), which mediates the cellular immune response to infected cells, contributing to the clearance of intracellular pathogens and infected cells (Gallichan and Rosenthal, 1996; Brighenti and Andersson, 2012). In this study, we determined the potential ability of rLM induced $\mathrm{CD}^{+}$cytotoxic lymphocytes activity. Tuberculin purified protein derivative (PPD) was used as stimulator for cytotoxicity assay, it has been verified by LC-MS/MS that Ag85B and ESAT-6 are the components of PPD (Borsuk et al., 2009). The result showed that elevated mycobacterial antigen-specific CTL immune responses were elicited by rLM delivering fusion antigen Ag85B-ESAT-6. It is well recognized that CTL activation correlates with the Th1 immune response (Huang et al., 2007). Additionally, significantly higher levels of pro-inflammatory cytokines IFN- $\gamma$, TNF- $\alpha$, IL-17, and IL6 were elicited by vaccinating with rLM, while lower levels of anti-inflammatory cytokine IL-4 was produced; on one hand, these pro-inflammatory factors play important roles in assisting and augmenting $\mathrm{CD}^{+}{ }^{+} \mathrm{T}$ cell immunity (Pamer, 2004); on the other hand, they improve the development of Th1 cells and contribute to the host response to mycobacteria. Among these pro-inflammatory cytokines, IFN- $\gamma$, and TNF$\alpha$ are considered to perform the major role of Th1 immune responses in protective immunity against $\mathrm{TB}(\mathrm{McNab}$ et al., 2015). IL-17 attracts the recruitment of Th1 cells to the site of infection, contributes antibacterial infection (Xu et al., 2010). IL- 6 producing by $\mathrm{T}$ cells and macrophages drives Th17 cells polarization, stimulates immune response, plays a role in fighting infection (Ladel et al., 1997). We consider that strong Th1 immune response, the strong $\mathrm{CD}^{+}$cytotoxic lymphocyte killing activity, heightened pro-inflammatory cytokines production and decreased anti-infammatory cytokines to have elevated the protection against high-dose M.tb $\mathrm{H} 37 \mathrm{Rv}$ challenge via the intravenous route.

Th17 cells, differentiated from naïve $\mathrm{CD}^{+} \mathrm{T}$ cells, are the major cell type producing IL-17A (Xu et al., 2010). The importance of IL-17A in inherent immunity is well known, while its importance in adaptive immunity and protective immunity for M.tb infection has not received enough attention until recent years (Khader and Gopal, 2010). Verwaerde's report found that a lack of significant IL-17 production in the spleen and lung resulted in no significant protection after HBHA vaccination (Verwaerde et al., 2014). T cell immunity after vaccination with M72/AS01 in South African adults showed 

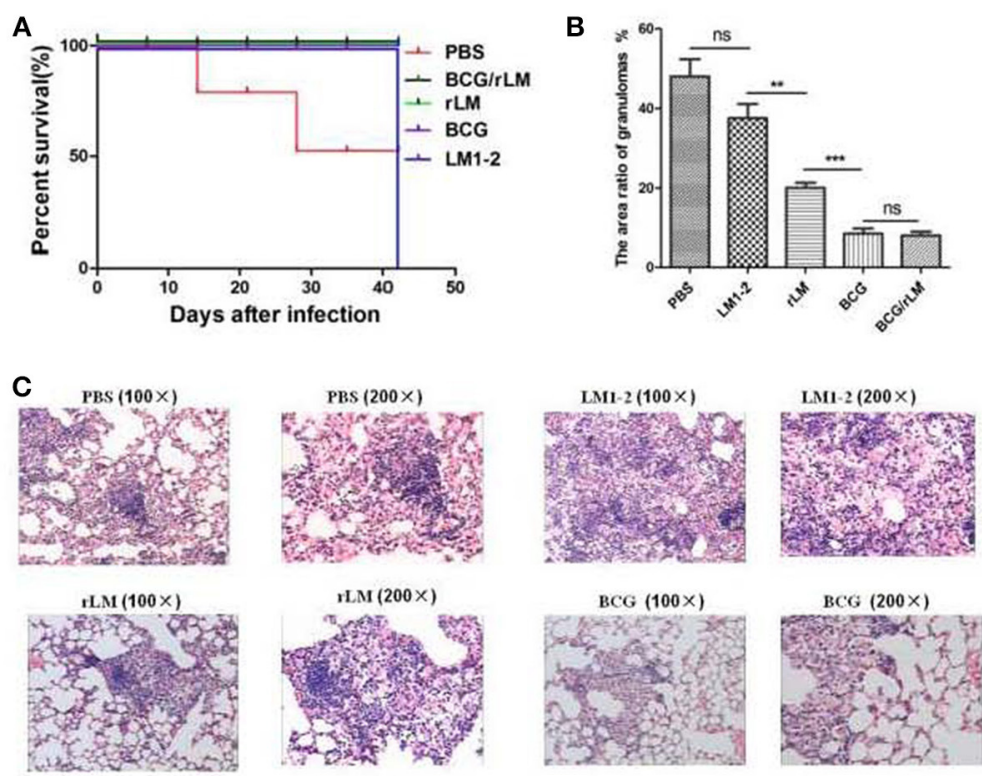

$\operatorname{BCG}(200 \times)$

BCG ILMA $(100 \times)$
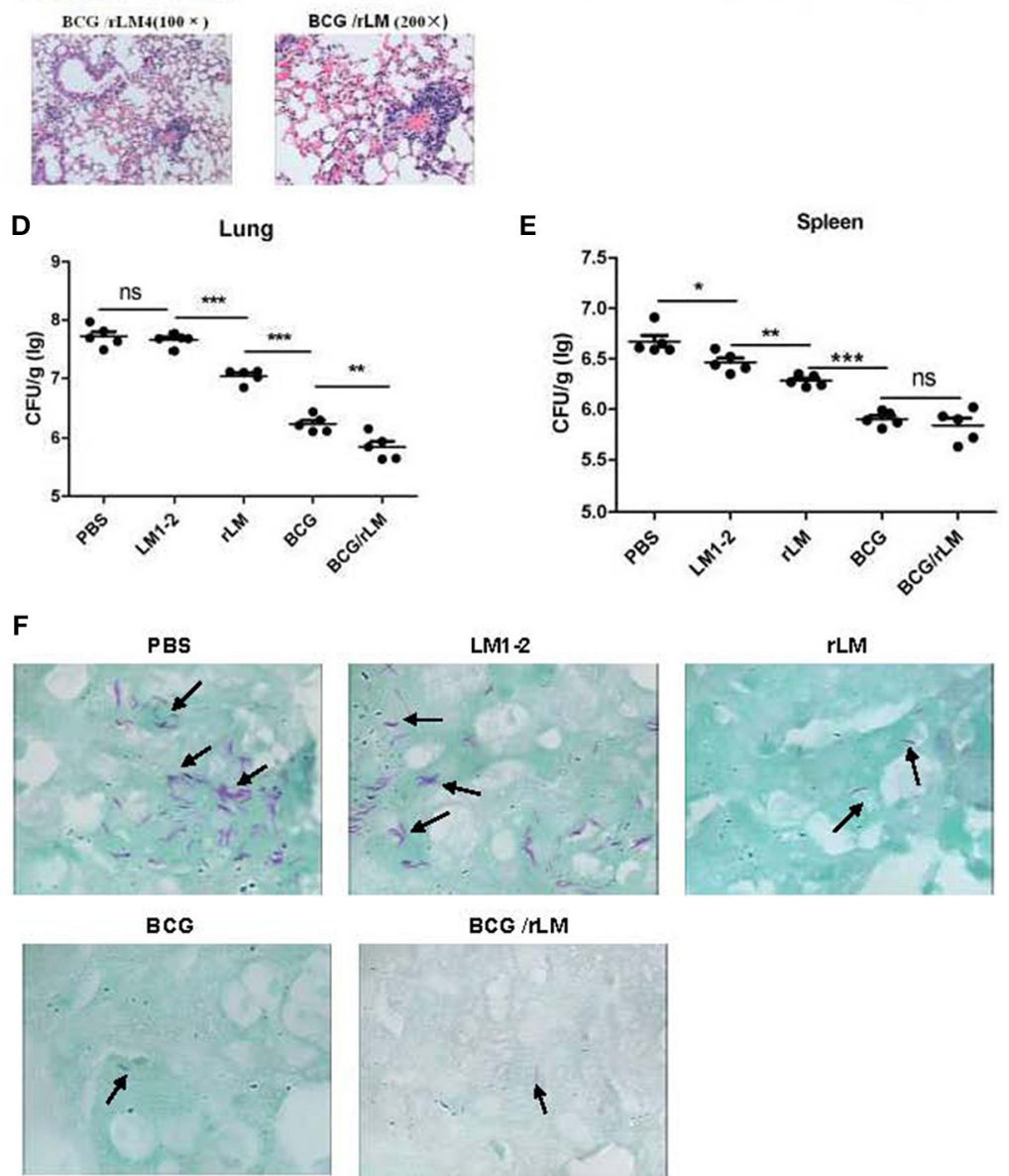

FIGURE 4 | Protective efficacy of prime-boost vaccination with rLM. After 42 days of immunization, mice were challenged with $5 \times 10^{5} \mathrm{CFU}$ virulent M.tb $\mathrm{H} 37 \mathrm{Rv}$ via a lateral tail vein. Six weeks post-challenge, all mice were sacrificed. The spleens and livers of five mice per group were removed, homogenized and cultured for CFU of M.tb. The left lung of each mouse was excised and fixed in 10\% phosphate-buffered formalin, sectioned, stained with hematoxylin and eosin, and examined for histological lesions with microscope. Alternatively, the tissues were subjected to acid-fast staining to visualize the bacilli. The challenge experiment was conducted twice. (A) Survival of the immunized mice challenged by M.tb H37Rv. (B) The area ratio of granulomas per slice in each group of mice; (C) Histopathology (HandE staining) of the spleen in mice in each group; (D,E) Bacteria load in lung (D) and spleen (E) of mice. (F) Lung tissue with anti-acid staining. 
that most vaccination-induced $\mathrm{T}$ cells did not express $\mathrm{Th} 1$ cytokine or IL-17 (Day et al., 2013). Gopal's study verified that early protective immunity against hypervirulent M.tb HN878 requires IL-17 (Gopal et al., 2014). Therefore, the Th17 immune response is an important goal for an effective vaccine against TB (Muranski et al., 2011). In this study, the IL-17A production levels of Th17 cells in the spleen of mice were determined, and the results indicated that BCG vaccination can't induce obviously IL-17 secretion in splenic lymphocyte stimulated with $\mathrm{PPD}$ or $\mathrm{FbpB}_{240-259}$, this result was consistent with Garcia-Pelayo's report (Garcia-Pelayo et al., 2015), while rLM could induce significantly FbpB-ESAT-6specific IL-17 ${ }^{+} \mathrm{T}$ cell immune responses, it is consistent with Xu's report of a Listeria vector delivering OVA which verified that Th17 cells promote the CTL response and that IL-17A is required for the generation of an Ag-specific CD8 ${ }^{+} \mathrm{T}$ cell response against primary infection (Xu et al., 2010). Based on the above-mentioned reports and our results, we deduce that Th1/Th17 immune responses elicited by vaccination with rLM were crucial for the reduction in the bacterial load and in the inflammatory lung lesions caused by M.tb challenge in C57BL/6/c mice and could afford $100 \%$ protection efficacy against M.tb H37Rv.

BCG strains lack numerous M.tb antigens, some epitopes sequence variants have not been maintained in the BCG strains, including epitopes derived from $f b p B, m c e$, and hemK (Copin et al., 2014). Moreover, T cell antigens such as ESAT-6, CFP10, and PPE68 are absent from BCG strains, which are the main reason for BCG's failure to induce sufficient cellular immune protection (Copin et al., 2014). Secretory proteins ESAT-6 and $\mathrm{FbpB}(\mathrm{Ag} 85 \mathrm{~B})$ with multiple $\mathrm{T}$ cell epitopes and $\mathrm{B}$ cell epitopes are crucial protective antigens (D'Souza et al., 2003; Brodin et al., 2006). In this study, the fusion protein FbpBESAT-6 was expressed and secreted by a Listeria vector and could induce strong Th1-type immune responses and confer efficient protection against high doses of $M . t b \mathrm{H} 37 \mathrm{Rv}$ intravenous infection. rLM could induce of Th1-type immune response, thus it is a novel attractive vaccine candidate with clear advantages. Finally, with minor details needing to be finalized, the peptide ESAT- $6_{1-20}$ stimulation did not generate the most robust immune responses comparing with $\mathrm{FbpB}_{240-259}$, it is possibly related with the construction strategy, ESAT- 6 was fused to FbpB with linker of Gly-Gly-Gly-Gly-Ser, the amino acid sequence is downstream of $\mathrm{FbpB}, 3 \mathrm{D}$ structure may be partly affected to the natural epitopes of ESAT-6. The optimization of order, linker, and copies of two antigens deserves further exploration to improve the induction of antigen specific cellular immunity.

Because novel vaccine candidates are intended to prevent human tuberculosis in clinical use, safety is a first consideration. Accordingly, we chose a highly attenuated serovar, 1/2a L. monocytogenes with deletion of actA (75-bp signal sequence maintained) and $p l c B$ as the parental strain. Its $50 \%$ lethal dose in mice was increased 46,700-fold compared to the parent strain. Moreover, the serotype of strain LM1-2 is the same as for attenuated Lm H1169 with deletion of $49 \%$ of act $A$ and $82 \%$ of plcB. A study of the safety of $\mathrm{H} 1169$ by Angelakopoulos et al demonstrated that actA/plcB-deleted attenuated LM may be administered without serious sequelae to carefully monitored adult volunteers (Angelakopoulos et al., 2002). The enhanced safety of this recombinant TB vaccine candidate lays a strong foundation for the evaluation of clinical efficacy. More importantly, our reports verified that the deletion of both act $A$ and $p l c B$ allows the induction of robust $\mathrm{T}$ cell responses in mouse models.

In summary, our data demonstrated that rLM remarkably increased the levels of Th1 cytokine and the activity of CTLs. Moreover, rLM could enhance the vaccination effects and significantly elevate protection against M.tb H37Rv challenge, e.g., reducing the bacterial burden and pathological lesions in the lungs and spleen. More importantly, the safety profile of the recombinant strain is encouraging. Collectively, our findings indicated that rLM could compensate the limitations of BCG, as a novel and useful tool for preventing M.tb infection, and a promising vaccine used to boost BCG-primed immunity.

\section{AUTHOR CONTRIBUTIONS}

YY and KL made main part of all experiments, and YY finished written the manuscript. DZ, CT, and WT took part in the immune analysis experiments. XC and XW participated in pathological observation. ZX and $\mathrm{MH}$ had a hand in analysis with flow cytometry. XiaohZ, ZP, and XiaomZ guided the implement of the experiments, XiaohZ also revised the manuscript, XiaomZ guided the operation related with labeled and analyzed with FACS. YR took part in challenge experiments. XJ guided the implement of all experiments, and supported the funds.

\section{FUNDING}

This work was supported by the National Key Research and Development Program of China (2017YFD0500300), the National Natural Science Foundation of China (No. 31472193, 31602031) and External Cooperation Program of BIC, CAS (No. GJHZ201312).

\section{ACKNOWLEDGMENTS}

We thank the staff of ABSL-3 Lab, for permitting and providing the conditions to carry out challenge experiments.

\section{SUPPLEMENTARY MATERIAL}

The Supplementary Material for this article can be found online at: http://journal.frontiersin.org/article/10.3389/fcimb. 2017.00407/full\#supplementary-material 


\section{REFERENCES}

Agnellini, P., Wiesel, M., Schwarz, K., Wolint, P., Bachmann, M. F., and Oxenius, A. (2008). Kinetic and mechanistic requirements for helping CD8 T cells. J. Immunol. 180, 1517-1525. doi: 10.4049/jimmunol.180.3.1517

Angelakopoulos, H., Loock, K., Sisul, D. M., Jensen, E. R., Miller, J. F., and Hohmann, E. L. (2002). Safety and shedding of an attenuated strain of Listeria monocytogenes with a deletion of act $\mathrm{A} / \mathrm{plcB}$ in adult volunteers: a dose escalation study of oral inoculation. Infect. Immun. 70, 3592-3601. doi: 10.1128/IAI.70.7.3592-3601.2002

Beverley, P. C., Sridhar, S., Lalvani, A., and Tchilian, E. Z. (2014). Harnessing local and systemic immunity for vaccines against tuberculosis. Mucosal Immunol. 7, 20-26. doi: 10.1038/mi.2013.99

Borsuk, S., Newcombe, J., Mendum, T. A., Dellagostin, O. A., and McFadden, J. (2009). Identification of proteins from tuberculin purified protein derivative (PPD) by LC-MS/MS. Tuberculosis 89, 423-430. doi: 10.1016/j.tube.2009.07.003

Brighenti, S., and Andersson, J. (2012). Local immune responses in human tuberculosis: learning from the site of infection. J. Infect. Dis. 205(Suppl. 2), S316-S324. doi: 10.1093/infdis/jis043

Brodin, P., Majlessi, L., Marsollier, L., de Jonge, M. I., Bottai, D., and Demangel, C. (2006). Dissection of ESAT-6 system 1 of Mycobacterium tuberculosis and impact on immunogenicity and virulence. Infect. Immun. 74, 88-98. doi: 10.1128/IAI.74.1.88-98.2006

Cavalcanti, Y. V., Brelaz, M. C., Neves, J. K., Ferraz, J. C., and Pereira, V. R. (2012). Role of TNF-Alpha, IFN-Gamma, and IL-10 in the development of pulmonary tuberculosis. Pulm. Med. 2012:745483. doi: 10.1155/2012/745483

Condotta, S. A., Richer, M. J., Badovinac, V. P., and Harty, J. T. (2012). Probing CD8 T cell responses with Listeria monocytogenes infection. Adv. Immunol. 113, 51-80. doi: 10.1016/B978-0-12-394590-7.00005-1

Copin, R., Coscollá, M., Efstathiadis, E., Gagneux, S., and Ernst, J. D. (2014). Impact of in vitro evolution on antigenic diversity of Mycobacterium bovis bacillus Calmette-Guerin (BCG). Vaccine 32, 5998-6004. doi: 10.1016/j.vaccine.2014.07.113

Day, C. L., Tameris, M., Mansoor, N., van Rooyen, M., de Kock, M., and Geldenhuys, H. (2013). Induction and regulation of T-cell immunity by the novel tuberculosis vaccine M72/AS01 in South African adults. Am. J. Respir. Crit. Care Med. 188, 492-502. doi: 10.1164/rccm.201208-1385OC

Decatur, A. L., and Portnoy, D. A. (2000). A PEST-like sequence in listeriolysin O essential for Listeria monocytogenes pathogenicity. Science 290, 992-995. doi: $10.1126 /$ science.290.5493.992

D’Souza, S., Rosseels, V., Romano, M., Tanghe, A., Denis, O., and Jurion, F. (2003). Mapping of murine Th1 helper T-cell epitopes of mycolyl transferases Ag85A, Ag85B, and Ag85C from Mycobacterium tuberculosis. Infect. Immun. 10, 483-493. doi: 10.1128/IAI.71.1.483-493.2003

Gallichan, W. S., and Rosenthal, K. L. (1996). Long-lived cytotoxic T lymphocyte memory in mucosal tissues after mucosal but not systemic immunization. J. Exp. Med. 184, 1879-1890. doi: 10.1084/jem.184.5.1879

Garcia-Pelayo, M. C., Bachy, V. S., Kaveh, D. A., Kaveh, D. A., and Hogarth, P. J. (2015). BALB/c mice display more enhanced BCG vaccine induced Th1 and Th17 response than C57BL/6 mice but have equivalent protection. Tuberculosis 95, 48-53. doi: 10.1016/j.tube.2014.10.012

Garg, N. K., Dwivedi, P., Jain, A., Tyagi, S., Sahu, T., and Tyagi, R. K. (2014). Development of novel carrier(s) mediated tuberculosis vaccine: more than a tour de force. Eur. J. Pharm. Sci. 62, 227-242. doi: 10.1016/j.ejps.2014. 05.028

Gopal, R., Monin, L., Slight, S., Uche, U., Blanchard, E., and Fallert Junecko, B. A. (2014). Unexpected role for IL-17 in protective immunity against hypervirulent Mycobacterium tuberculosis HN878 infection. PLoS Pathog. 10:e1004099. doi: 10.1371/journal.ppat.1004099

Grode, L., Seiler, P., Baumann, S., Hess, J., Brinkmann, V., and Eddine, A. N. (2005). Increased vaccine efficacy against tuberculosis of recombinant Mycobacterium bovis bacille Calmette-Guérin mutants that secrete listeriolysin. J. Clin. Invest. 115, 2472-2479. doi: 10.1172/JCI24617

Gunn, G. R., Zubair, A., Peters, C., Pan, Z. K., Wu, T. C., and Paterson, Y. (2001). Two Listeria monocytogenes vaccine vectors that express different molecular forms of human papilloma virus-16 (HPV-16) E7 induce qualitatively different $\mathrm{T}$ cell immunity that correlates with their ability to induce regression of established tumors immortalized by HPV-16. J. Immunol. 167, 6471-6479. doi: 10.4049/jimmunol.167.11.6471

Henao-Tamayo, M., Ordway, D. J., and Orme, I. M. (2014). Memory T cell subsets in tuberculosis: what should we be targeting? Tuberculosis 94, 455-461. doi: 10.1016/j.tube.2014.05.001

Huang, H., Hao, S., Li, F., Ye, Z., Yang, J., and Xiang, J. (2007). CD4+ Th1 cells promote $\mathrm{CD}^{+} \mathrm{Tc} 1$ cell survival, memory response, tumor localization and therapy by targeted delivery of interleukin 2 via acquired pMHC I complexes. Immunology 120, 148-159. doi: 10.1111/j.1365-2567.2006.02452.x

Jiang, S., Rasmussen, R. A., Nolan, K. M., Frankel, F. R., Lieberman, J., and McClure, H. M. (2007). Live attenuated Listeria monocytogenes expressing HIV Gag: immunogenicity in rhesus monkeys. Vaccine 25, 7470-7479. doi: 10.1016/j.vaccine.2007.08.013

Khader, S. A., and Gopal, R. (2010). IL-17 in protective immunity to intracellular pathogens. Virulence 1, 423-427. doi: 10.4161/viru.1.5.12862

Kim, C., Jay, D. C., and Williams, M. A. (2014). Dynamic functional modulation of $\mathrm{CD} 4+\mathrm{T}$ cell recall responses is dependent on the inflammatory environment of the secondary stimulus. PLoS Pathog. 10:e1004137. doi: 10.1371/journal.ppat.1004137

Kollmann, T. R., Reikie, B., Blimkie, D., Way, S. S., Hajjar, A. M., and Arispe, K. (2007). Induction of protective immunity to Listeria monocytogenes in neonates. J. Immunol. 178, 3695-3701. doi: 10.4049/jimmunol.178.6.3695

Kono, M., Nakamura, Y., Suda, T., Uchijima, M., Tsujimura, K., and Nagata, T. (2012). Enhancement of protective immunity against intracellular bacteria using type-1 polarized dendritic cell (DC) vaccine. Vaccine 30, 2633-2639. doi: 10.1016/j.vaccine.2012.02.026

Ladel, C. H., Blum, C., Dreher, A., Reifenberg, K., Kopf, M., and Kaufmann, S. H. (1997). Lethal tuberculosis in interleukin-6-deficient mutant mice. Infect. Immun. 65, 4843-4849.

Lin, Q., Zhou, M., Xu, Z., Khanniche, A., Shen, H., and Wang, C. (2015). Construction of two Listeria ivanovii attenuated strains expressing Mycobacterium tuberculosis antigens for TB vaccine purposes. J. Biotechnol. 196-197, 20-26. doi: 10.1016/j.jbiotec.2015.01.008

Maciag, P. C., Radulovic, S., and Rothman, J. (2009). The first clinical use of a liveattenuated Listeria monocytogenes vaccine: a phase I safety study of Lm-LLO-E7 in patients with advanced carcinoma of the cervix. Vaccine 27, 3975-3983. doi: 10.1016/j.vaccine.2009.04.041

McNab, F., Mayer-Barber, K., Sher, A., Wack, A., and O'Garra, A. (2015). Type I interferons in infectious disease. Nat. Rev. Immunol. 15, 87-103. doi: $10.1038 /$ nri3787

Miki, K., Nagata, T., Tanaka, T., Kim, Y. H., Uchijima, M., and Ohara, N. (2004). Induction of protective cellular immunity against Mycobacterium tuberculosis by recombinant attenuated self-destructing Listeria monocytogenes strains harboring eukaryotic expression plasmids for antigen 85complex and MPB/MPT51. Infect. Immun. 72, 2014-2021. doi: 10.1128/IAI.72.4.2014-2021.2004

Muranski, P., Borman, Z. A., Kerkar, S. P., Klebanoff, C. A., Ji, Y., and SanchezPerez, L. (2011). Th17 cells are long lived and retain a stem cell-like molecular signature. Immunity 35, 972-985. doi: 10.1016/j.immuni.2011.09.019

Nomura, T., Kawamura, I., Tsuchiya, K., Kohda, C., Baba, H., and Ito, Y. (2002). Essential role of interleukin-12 (IL-12) and IL-18 for gamma interferon production induced by listeriolysin $\mathrm{O}$ in mouse spleen cells. Infect. Immun. 70, 1049-1055. doi: 10.1128/IAI.70.3.1049-1055.2002

Pamer, E. G. (2004). Immune responses to Listeria monocytogenes. Nat. Rev. Immunol. 4, 812-823. doi: 10.1038/nri1461

Petit, R. G., and Basu, P. (2013). ADXSII0001 LM-LLO immunotherapy targeting HPVE7: preliminary safety and durvival data from a phase 2 study in Indian women with recurrent/refractory cervical cancer. J. Immunother. Cancer 1:P231. doi: 10.1186/2051-1426-1-S1-P231

Principi, N., and Esposito, S. (2015). The present and future of tuberculosis vaccinations. Tuberculosis 95, 6-13. doi: 10.1016/j.tube.2014.10.004

Rook, G. A., Hernandez-Pando, R., Dheda, K., and Teng Seah, G. (2004). IL-4 in tuberculosis: implications for vaccine design. Trends Immunol. 2, 483-488. doi: 10.1016/j.it.2004.06.005

Shang, S., Siddiqui, S., Bian, Y., Zhao, J., and Wang, C. R. (2016). Nonclassical MHC Ib-restricted CD8 ${ }^{+} \mathrm{T}$ cells recognize Mycobacterium tuberculosis-derived protein antigens and contribute to protection against infection. PLoS Pathog. 12:e1005688. doi: 10.1371/journal.ppat.1005688 
Torrado, E., and Cooper, A. M. (2010). IL-17 and Th17 cells in tuberculosis. Cytokine Growth Factor Rev. 21, 455-462. doi: 10.1016/j.cytogfr.2010. 10.004

Verwaerde, C., Debrie, A. S., Dombu, C., Legrand, D., Raze, D., and Lecher, S. (2014). HBHA vaccination may require both Thl and Th17 immune responses to protect mice against tuberculosis. Vaccine 32, 6240-6250. doi: 10.1016/j.vaccine.2014.09.024

Watanabe-Takano, H., Takano, K., Sakamoto, A., Matsumoto, K., Tokuhisa, T., and Endo, T. (2014). DA-Raf-dependent inhibition of the Ras-ERK signaling pathway in type 2 alveolar epithelial cells controls alveolar formation. Proc. Natl. Acad. Sci. U.S.A. 111, E2291-E2300. doi: 10.1073/pnas.13215 74111

World Health Organization (2016). Tuberculosis. Available online at: http://www.who.int/mediacentre/factsheets/fs104/en/

Xu, S., Han, Y., Xu, X., Bao, Y., Zhang, M., and Cao, X. (2010). IL17A-producing gammadelta $\mathrm{T}$ cells promote $\mathrm{CTL}$ responses against Listeria monocytogenes infection by enhancing dendritic cell crosspresentation. J. Immunol. 185, 5879-5887 doi: 10.4049/jimmunol. 1001763

Yamamoto, K., Kawamura, I., Tominaga, T., Nomura, T., Kohda, C., and Ito, J. (2005). Listeriolysin, O., a cytolysin derived from Listeria monocytogenes inhibits generation of ovalbumin-specific Th2 immune response by skewing maturation of antigen-specific T cells into Th1 cells. Clin. Exp. Immunol. 142, 268-274. doi: 10.1111/j.1365-2249.2005.02922.x

Yin, Y., Tian, D., Jia, Y., Gao, Y., Fu, H., and Niu, Z. (2012). Attenuated Listeria monocytogenes, a Mycobacterium tuberculosis ESAT-6 antigen expression and delivery vector for inducing an immune response. Res. Microbiol. 163, 540-549. doi: 10.1016/j.resmic.2012.07.008

Yin, Y., Zhu, G., Geng, S., Hu, M., and Jiao, X. (2008). Construction and characterization of a mutant strain of Listeria monocytogenes with a deletion of actA and plcB. Wei Sheng Wu Xue Bao 48, 299-303. doi: 10.13343/j.cnki.wsxb.2008.03.004

Conflict of Interest Statement: The authors declare that the research was conducted in the absence of any commercial or financial relationships that could be construed as a potential conflict of interest.

Copyright (C) 2017 Yin, Lian, Zhao, Tao, Chen, Tan, Wang, Xu, Hu, Rao, Zhou, Pan, Zhang and Jiao. This is an open-access article distributed under the terms of the Creative Commons Attribution License (CC BY). The use, distribution or reproduction in other forums is permitted, provided the original author(s) or licensor are credited and that the original publication in this journal is cited, in accordance with accepted academic practice. No use, distribution or reproduction is permitted which does not comply with these terms. 